\title{
Long noncoding RNA SFTA1P promoted apoptosis and increased cisplatin chemosensitivity via regulating the hnRNP-U-GADD45A axis in lung squamous cell carcinoma
}

\author{
Ling Li ${ }^{1,2}$, Ji-Ye Yin ${ }^{1,2}$, Fa-Zhong $\mathrm{He}^{1,2}$, Ma-Sha Huang ${ }^{1,2}$, Tao Zhu ${ }^{1,2}$, Yuan-Feng \\ Gao $^{1,2}$, Yi-Xin Chen ${ }^{1,2}$, Dong-Bo Zhou ${ }^{3}$, Xiang Chen ${ }^{4}$, Lun-Quan Sun ${ }^{5}$, Wei Zhang ${ }^{1,2}$, \\ Hong-Hao Zhou ${ }^{1,2}$ and Zhao-Qian Liu ${ }^{1,2}$ \\ ${ }^{1}$ Department of Clinical Pharmacology, Xiangya Hospital, Central South University, Changsha 410008, P. R. China \\ ${ }^{2}$ Institute of Clinical Pharmacology, Hunan Key Laboratory of Pharmacogenetics, Central South University, Changsha 410078, \\ P. R. China \\ ${ }^{3}$ Department of Gerontology, Xiangya Hospital, Central South University, Changsha 410008, P. R. China \\ ${ }^{4}$ Department of Dermatology, Xiangya Hospital, Central South University, Changsha 410008, P. R. China \\ ${ }^{5}$ Center for Molecular Medicine, Xiangya Hospital, Key Laboratory of Molecular Radiation Oncology of Hunan Province, Central \\ South University, Changsha 410008, P. R. China \\ Correspondence to: Zhao-Qian Liv, email: livzhaoqian63@126.com \\ Keywords: lung squamous cell carcinoma; long noncoding RNA SFTA IP; hnRNP-U- GADD45A; apoptosis; cisplatin chemosensitivity \\ Received: July 28, 2017 \\ Accepted: August 24, 2017 \\ Published: October 27, 2017 \\ Copyright: Li et al. This is an open-access article distributed under the terms of the Creative Commons Attribution License 3.0 (CC \\ BY 3.0), which permits unrestricted use, distribution, and reproduction in any medium, provided the original author and source are \\ credited.
}

\section{ABSTRACT}

Chemotherapeutic insensitivity remains one of the major obstacles in clinical treatment of lung squamous cell carcinoma (LSCC). Recently, increasing evidence has suggested that long non-coding RNAs (IncRNAs) promote tumorigenesis in many cancer types. However, the potential biological roles and regulatory mechanisms of IncRNAs in response to cisplatin treatment are poorly understood. Here, we found that IncRNA SFTA1P (surfactant associated 1, pseudogene), highly expressed in lung, was down-regulated in LSCC tissues and could be induced upon cisplatin treatment in LSCC cells. Elevated SFTA1P induced apoptosis and enhanced the sensitivity to cisplatin of LSCC cells. We further identified that hnRNP-U (heterogeneous nuclear ribonucleoprotein $U$ ) was down-regulated in LSCCs and positively correlated with patients' poor prognosis as well as SFTA1P. Mechanistic studies revealed that SFTA1P could up-regulate hnRNP-U expression. In addition, we identified that hnRNP-U enhanced cisplatin-induced apoptosis through up-regulation of GADD45A, high expression of which was correlated with good prognosis in LSCC patients. Our findings demonstrated that SFTA1P might serve as a useful biomarker for LSCC diagnosis and a predictor for cisplatin chemotherapy response in patients with LSCC.

\section{INTRODUCTION}

According to the GLOBOCAN project of the World Health Organization in 2012, lung cancer remains the top cancer killer worldwide (http://globocan.iarc. $\mathrm{fr} /$ ). Lung squamous cell carcinoma (LSCC) is the second most common histologic subtype of lung cancer (preceded only by adenocarcinoma), accounting for $40 \sim 50 \%$ of all primary lung cancers [1]. As opposed to adenocarcinoma, genomic alterations in LSCC have not been comprehensively characterized and no specific molecularly targeted therapies have been developed for the treatment of LSCC [1]. And unfortunately, targeted agents developed for lung adenocarcinoma are largely ineffective against LSCC. Currently, surgical resection is the primary treatment approach for early-stage LSCC [2]. While in locally advanced unresectable or metastatic LSCC, cisplatin-based chemotherapy remains the cornerstone of 
the front-line systemic treatment $[3,4]$. Despite multiple options for the treatment of LSCC, the overall 5-year survival rate has not been improved significantly [5]. Chemotherapy resistance, either intrinsic or acquired, is a major clinical problem in LSCC, owing to individual variations in response to cisplatin based chemotherapy [3]. Therefore, understanding the molecular mechanisms underlying tumorigenesis and chemotherapy resistance is crucial for the development of effective therapeutic strategies for LSCC.

Long non-coding RNAs (lncRNAs) are transcripts longer than 200 nucleotides [8] which were once considered as transcriptional "noise" without biological function [6, 7]. However, the definition of lncRNA is controversial, recent research has shown that some lncRNAs can encode short peptides [9]. Accumulating evidence indicates that the majority of lncRNAs play key roles in cancer therapy through influencing cell cycle regulation, survival, cheomothrapy response, and various biological processes by modulating gene expression at the transcriptional, posttranscriptional and epigenetic regulation levels [10-14]. The majority $(\sim 78 \%)$ of lncRNAs are characterized by their tissuespecific, developmental stage specific expression mode, in contrast to expression of mRNAs with only $\sim 19 \%$ of them with tissue specificity $[15,16]$. Dysregulation of lncRNAs contributes to cancer progression, and they are therefore considered as potential therapeutic targets [17]. Our previous study found that deregulation of a panel of lncRNAs was involved in LSCC, based on a high throughput microarray screening [18]. Among them, SFTA1P was identified significantly downregulated in LSCC tissues, as compared with paired adjacent normal tissues.

In the current study, we focused on the function and regulatory mechanism of SFTA1P in LSCC. SFTA1P is a pseudogene-derived long non-coding RNA. By analyzing the relationship between SFTA1P and LSCC patients' clinical features and survival, we found that decreased SFTA1P expression was correlated with poor prognosis. Further mechanistic studies revealed that SFTA1P could bind with and upregulate hnRNP-U. HnRNP-U, also named scaffold attachment factor (SAF)-A is involved in mRNA processing and transporting [19-21]. For instance, hnRNP-U can enhance the expression of GADD45A by stabilizing mRNA [20]. More recent studies have documented that hnRNP-U was involved in cell apoptosis [22], DNA damage response [23]. In this study, we identified that SFTA1P enhanced cisplatin-induced apoptosis through regulation of the hnRNP-U-GADD45A pathway. SFTA1P is an essential regulator in cisplatin induced apoptosis, and the SFTA1P-hnRNP-U-GADD45A signaling axis plays an important role in increasing LSCC chemosensitivity. In addition, knockdown of hnRNP-U also contributes to cisplatin resistance through decreasing the expression of the apoptosis and DNA repair related gene GADD45A, similar to SFTA1P depletion induced effects. Thus, we concluded that the lncRNA SFTA1P may enhance cisplatin sensitivity in LSCC by increasing the expression of hnRNP-U and further facilitating GADD45A expression.

\section{RESULTS}

\section{Characterization of long non-coding RNA SFTA1P}

Long non-coding RNA SFTA1P is poly (A)-negative and located on chromosome 10 in humans. It consisted of four exons, with a full length of $873 n$ t (Figure 1A). Its coding-potential was evaluated by analysis of the sequences using ORF Finder from the National Center for Biotechnology Information and it failed to predict a protein of more than 58 amino acids (Figure 1B). Moreover, lncRNA SFTA1P does not contain a valid Kozak sequence. In addition, we used txCdsPredict from UCSC and PhyloCSF [24] to calculate its coding potential, it further supported that lncRNA SFTA1P has very little protein-coding potential (Figure 1C).

It is well known that the subcellular localization will affect the lncRNA function in cells. Next, we employed an established fluorescence in situ hybridization (FISH) assay to observe SFTA1P localization in NCl-H226 and A549 cells by confocal laser scanning microscope. We found that SFTA1P was mainly located in the nucleus in both cell lines (Figure 1E). And this result was further verified by cytoplasmic and nuclear RNA fractionation analysis (Figure 1F). More importantly, according to RNA-Seq gene expression profiles from BioGPS Database [25], we found that lncRNA SFTA1P was specifically highly expressed in the lung (Figure 1D).

\section{The low expression of LncRNA SFTA1P was correlated with poor prognosis in LSCC patients}

In our previous study [18], we found that SFTA1P was downregulated in 16 LSCC tissues than in the paired adjacent normal tissues detected by microarray (Figure 2A). The result was further validated by qRTPCR in another independent cohort of 80 paired LSCC tissues (Figure 2B). In addition, we analyzed the SFTA1P expression level in common LSCC cell lines (NCl-H226, SK-MES-1), lung adenocarcinoma (LUAD) cell lines (NCl-H1299, A549, A549-DDP) and a normal bronchial epithelial cell line (16HBE). Also, we found that SFTA1P was significantly down-regulated in both LSCC and LUAD cell lines than in 16HBE cells (Figure 2C). Of note, we found decreased expression of SFTA1P in A549 cisplatin resistant cells (A549-DDP) than in the parental A549 cell line (Figure 2C). These data indicated that SFTA1P may play a role in tumorigenesis and cisplatin sensitivity in lung cancer. 
Meantime, we further examined whether SFTA1P expression was correlated with LSCC patients' clinical pathological features using univariate analysis. The patients were divided into two groups: the high-SFTA1P group $(\mathrm{n}=40)$ and the low-SFTA1P group $(\mathrm{n}=40)$, using the median of SFTA1P as the cutoff. Statistical analysis revealed that decreased $S F T A 1 P$ expression was correlated with advanced pathological stage $(\mathrm{OR}=2.38 \quad(0.91$, 6.26), $\mathrm{p}=0.077)$ and smoking status $(\mathrm{OR}=5.22$ (1.46, 18.68), $\mathrm{p}=0.011)$. However, SFTA1P expression was not associated with other factors including age $(\mathrm{OR}=0.98$ (0.92, 1.05), $\mathrm{p}=0.648)$, gender $(\mathrm{OR}=1.41(0.07,28.51)$, $\mathrm{p}=0.819)$, differentiation $\quad(\mathrm{OR}=0.726 \quad(0.13,3.97)$, $\mathrm{p}=0.712 ; \mathrm{OR}=0.802(0.22,2.86), \mathrm{p}=0.734)$ and lymph node metastasis $(\mathrm{OR}=1.34(0.51,3.05), \mathrm{p}=0.555)$ in LSCC (Table 1). Next, Kaplan-Meier survival analysis was performed to explore the relationship between SFTA1P expression and LSCC patient's survival based on data from a GEO dataset (GSE50081). The results showed that the overall survival (OS) and progression-free survival (PFS) rate over 10 years for the low-SFTA1P group is shorter than that in the high-SFTA1P group (Figure 2D and $2 \mathrm{E}$ ). These results demonstrated that lncRNA SFTA1P may serve as a biomarker to predict the prognosis of LSCC patients.

\section{LncRNA SFTA1P decreased cells viability and induced cell apoptosis}

To assess the biological function of SFTA1P on LSCC phenotype, we performed gain of-function assays to investigate the influence of SFTA1P on cell proliferation,

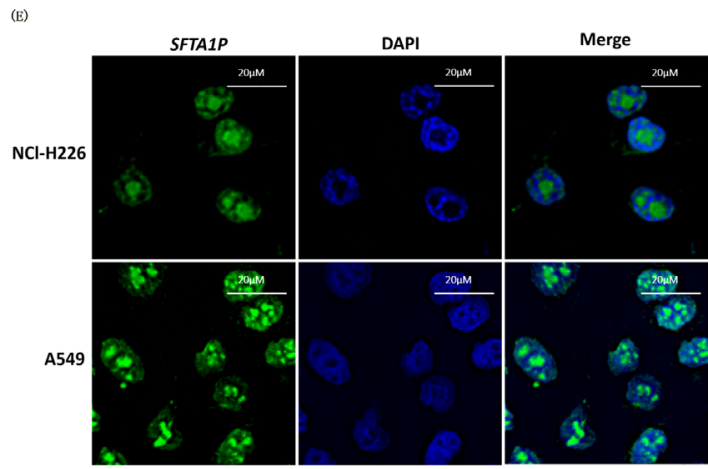

(F)

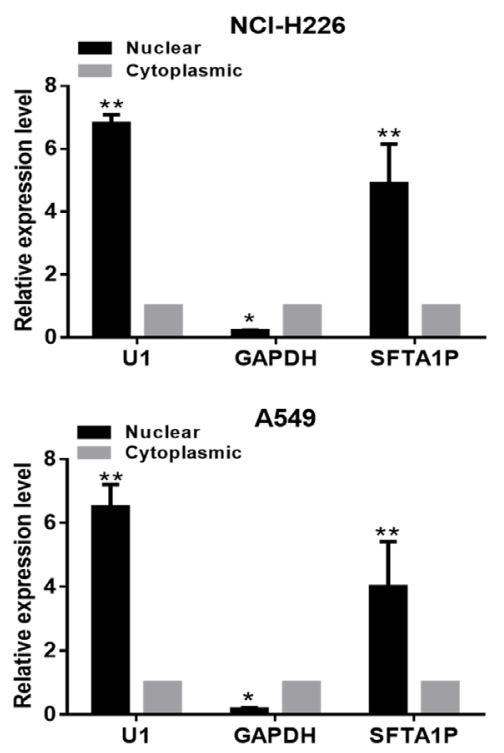

Figure 1: Characterization of SFTA1P. Panel A. Schematic annotation of SFTA1P genomic locus on chromosome 10q10.826.40210.836.877 in humans. Green rectangles represent exons. Panel B and Panel C. SFTA1P coding function analysis. Panel B. Analysis of the sequences by ORF Finder from the National Center for Biotechnology Information. Panel C. Used txCdsPredict from UCSC and PhyloCSF to calculate its coding potential. Panel D. SFTA1P specific expression in lung tissue. Panel E. Confocal FISH images showing nucleus localization of SFTA1P in NCI-H226 and A549 cells. Green represents SFTA1P; blue represent nucleus. Panel F. The RNA level of SFTA1P in nuclear and cytoplasmic fraction was determined by RT-PCR inNCI-H226 and A549 cells, respectively. U1 was a positive control for nuclear fraction and GAPDH was a positive control for cytoplasmic fraction. All experiments were performed in three biological replicates $(\mathrm{n}=3)$. And all data are shown as mean $\left.\pm \mathrm{SD},{ }^{*} \mathrm{p}<0.05,{ }^{* *} \mathrm{p}<0.05\right)$. 
apoptosis by transfecting PCDNA3.1(+)-SFTA1P vector into the LSCC cell lines (NCl-H226, SK-MES-1). Cells transfected with PCDNA3.1(+)-SFTA1P vector showed significantly increased SFTA1P expression in NCl-H226 and SK-MES-1 cells compared with that of the control group (Figure 3A). The MTS assay showed that the cell viability was decreased in SFTA1P overexpressing NCl-H226 and SK-MES-1 cells (Figure 3B). Oppositely, knockdown of SFTA1P by transfecting SFTA1P-specific siRNA (Figure 3C) led to increased cell viability (Figure 3D). Collectively, these results suggested that SFTA1P had a suppressive effect on LSCC cell viability possibly through inhibiting cell proliferation or inducing cell apoptosis.

EdU, an indicator of DNA synthesis, was used to detect the effect of SFTA1P on cell proliferation. Results showed that there was no significant difference between the percentage of EdU positive cells in the SFTA1P overexpression group and that of the control group (Figure $3 \mathrm{E})$. In addition, we detected the cell proliferation marker protein $\mathrm{Ki}-67$ expression level in NCl-H226 and SK-MES-1 cells, it also showed the same results (Figure 3F). These results suggest that SFTA1P has no effect on LSCC cell proliferation. So, we guessed that SFTA1P decreased cell viability probably via inducing cell apoptosis.

Then, we performed flow cytometric analysis to detect whether SFTA1P inducing cell apoptosis. Our results showed that the fraction of apoptotic cells was significantly increased in the SFTAIP overexpressing cells (Figure 3G). And, these results were further verified by Western blot analysis of cell apoptosis marker protein cleaved caspase 3 (Figure $3 \mathrm{H}$ ). It confirmed that SFTA1P was involved in the apoptosis of LSCC cells.

\section{Association of LncRNA SFTA1P with cell apoptosis and cisplatin-sensitivity}

As illustrated in Figure 2C, SFTA1P expression was downregulated in cisplatin resistant lung adenocarcinoma cell line A549/DDP, as compared with that in the parental cell line A549, suggesting that SFTA1P may involve in cisplatin chemoresistance for lung cancer.

In order to explore whether SFTA1P is essential for cisplatin chemosensitivity in LSCC, we determined the SFTA1P expression levels either at different time points in NCl-H226 and SK-MES-1 cells treated with $15 \mu \mathrm{M}$ cisplatin (Figure $4 \mathrm{~A}$ ) or $48 \mathrm{~h}$ after cells were treated with cisplatin at the indicated doses (Figure 4B). Interestingly, we observed that cisplatin induced SFTA1P expression in a time-dependent and dose-dependent manner. Meanwhile, we noticed that the cell apoptosis was also increased in a dose-dependent manner by detecting the apoptosis marker protein cleaved caspase 3 (Figure 4C). These data indicated that SFTA1P may involve in cisplatin chemosensitivity in LSCC.

Furthermore, cisplatin sensitivity, we performed MTS and flow cytometry assays to assess cell viability and apoptosis upon cisplatin treatment, respectively. Flow cytometric analyses using PE and Annexin V7-ADD
(A)

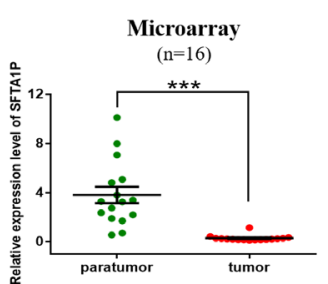

(C)

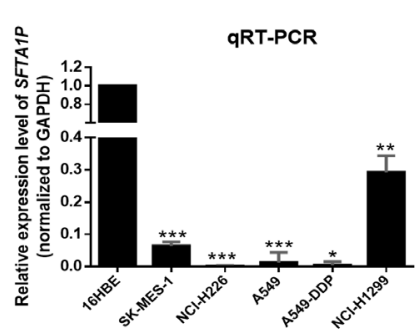

(B)

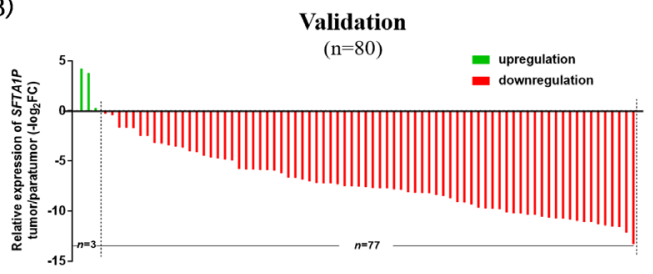

(D)

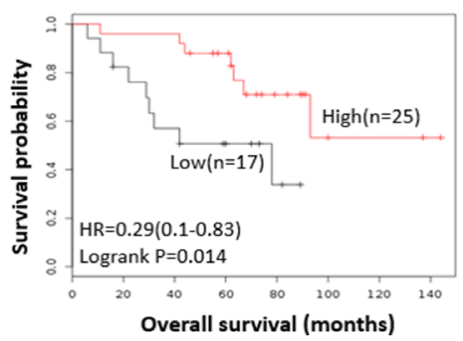

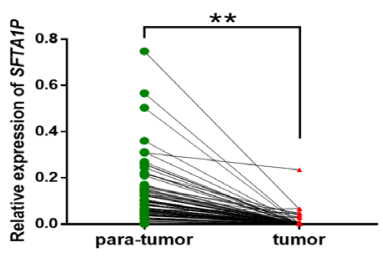

(E)

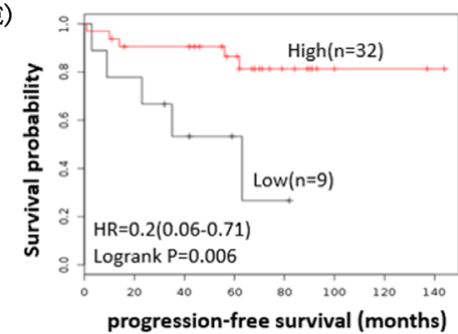

Figure 2: The expressions of LncRNA SFTA1P in LSCC cell lines and tissues as well as its potential clinical significance. Panel A-D. Relative expressions of the SFTA1P in LSCC tissues and cells and its clinical significance. Panel A. The expression level of SFTA1P in LSCC patient's para-tumor and tumor tissues was determined by microarray (n=16). Panel B. The expression level of SFTA1P was independent validation in 80 LSCC patient's para-tumor and tumor tissues by qRT-PCR. Panel C. qRT-PCR analysis of SFTA1P expression level in a normal human bronchial epithelial cell HBE and five common human NSCLC cell lines, NCl-H226, SK-MES-1, H1299, A549 and A549-DDP (cisplatin resistance cell line). ${ }^{*} \mathrm{p}<0.05,{ }^{* *} \mathrm{p}<0.01,{ }^{* * *} \mathrm{p}<0.001$. Panel D-E. Kaplan-Meier survival curve of LSCC patients with low levels of SFTA1P expression were correlated with a poor (D)overall survival and (E)progression-free survival. The median SFTA1P expression was used as a cutoff (data from GSE50081). 
Table 1: Correlation between SFTA1P expression and clinicopathological characteristics in 80 LSCC patients

\begin{tabular}{|c|c|c|c|c|c|}
\hline \multirow{2}{*}{ Clinical and pathological variables } & \multirow{2}{*}{$\mathbf{N}(\%)$} & \multicolumn{2}{|c|}{ SFTA1P expression levels } & \multirow{2}{*}{$O R(95 \% C I)$} & \multirow{2}{*}{$p$} \\
\hline & & High expression & Low expression & & \\
\hline Age (years) & & & & $0.98(0.92,1.05)$ & 0.648 \\
\hline$<60$ & $37(46.25)$ & 22 & 29 & & \\
\hline$\geq 60$ & $43(53.75)$ & 18 & 11 & & \\
\hline Gender & & & & $1.41(0.07,28.51)$ & 0.819 \\
\hline Male & $77(96.25)$ & 38 & 39 & & \\
\hline Female & $3(3.75)$ & 2 & 1 & & \\
\hline Smoking status & & & & $5.22(1.46,18.68)$ & 0.011 \\
\hline Smoker & $63(78.75)$ & 28 & 34 & & \\
\hline Non-smoker & $17(21.25)$ & 12 & 6 & & \\
\hline Clinical stage & & & & $2.38(0.91,6.26)$ & 0.077 \\
\hline I-II & $49(61.25)$ & 20 & 29 & & \\
\hline III-IV & $31(38.75)$ & 20 & 11 & & \\
\hline \multicolumn{6}{|l|}{ Differentiation } \\
\hline high & $13(16.25)$ & 7 & 6 & & \\
\hline Moderatly & $54(67.50)$ & 27 & 27 & $0.726(0.13,3.97)$ & 0.712 \\
\hline Poorly & $13(16.25)$ & 6 & 7 & $0.802(0.22,2.86)$ & 0.734 \\
\hline Lymph node metastasis & & & & $1.34(0.51,3.05)$ & 0.555 \\
\hline Yes & $35(43.75)$ & 20 & 15 & & \\
\hline No & $45(56.25)$ & 20 & 25 & & \\
\hline
\end{tabular}

double staining showed that when cells were treated with $15 \mu \mathrm{M}$ cisplatin for $24 \mathrm{~h}$, the fraction of apoptotic cells was notably increased in SFTA1P-overexpressing NCl-H226 cells compared with cells transfected with empty vector (Figure 4D).

$\mathrm{IC}_{50}$ value for cisplatin of cells overexpressing SFTA1P or with SFTA1P depletion was measured. Compared with the control groups, the $\mathrm{IC}_{50}$ value for cisplatin in NCl-H226 (or SK-MES-1) transfected with SFTA1P expressing vector was significantly reduced (Figure 4E and 4F). Conversely, compared with NCl-H226 (or SK-MES-1) cells transfected with siRNA-NC, the IC50 value for cisplatin in NCl-H226 or SK-MES-1 transfected with SFTA1P-specific siRNA was significantly increased (Figure $4 \mathrm{G}$ and $4 \mathrm{H}$ ). These results demonstrated that LncRNA SFTA1P enhanced sensitivity of LSCC cell lines to cisplatin.

Further, to confirm the role of SFTA1P, we used RNA fluorescence in situ hybridization (FISH) analysis to detect SFTA IP expression levels in 16 formalin-fixed paraffin-embedded (FFPE) LSCC tissues from patients treated with cisplatin-based chemotherapy. Eight patients experienced PR (partial response) and eight patients experienced PD (progressive disease) according to the RECIST criteria (Response Evaluation Criteria in Solid
Tumors). As shown in Figure 4I, SFTA1P expression was significantly higher in the PR patients than in the PD patients. These results demonstrated that lncRNA SFTA1P was possibly involved in the chemoresistance to cisplatin.

\section{LncRNA SFTA1P upregulated the expression of hnRNP-U}

We further explored the potential mechanism how SFTA1P influence cisplatin-induced apoptosis and regulate cisplatin resistance in LSCC. Through analyzing the data from a previous study (GSE34993) [26], we found that SFTA1P binded with heterogeneous nuclear ribonucleoprotein U (hnRNP-U, also called scaffold attachment factor A (SAF-A)) based on crosslinking and immunoprecipitation coupled with high-throughput sequencing (CLIP-seq) ( $\mathrm{p}=1.12 \mathrm{E}-44$, hypergeometric test; Figure 5A). We next examined RNA expression levels of SFTA1P and hnRNP-U in LSCC and adjacent normal tissues $(n=80)$. As shown in Figure $5 B$ and $5 C$, hnRNP-U expression was reduced in LSCC tissues compared with tissues. And further Kaplan-Meier survival analysis showed that LSCC patients with high hnRNP-U expression had notably longer overall survival (OS) and 
(A)

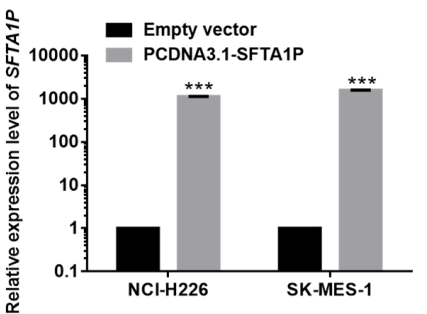

(C)

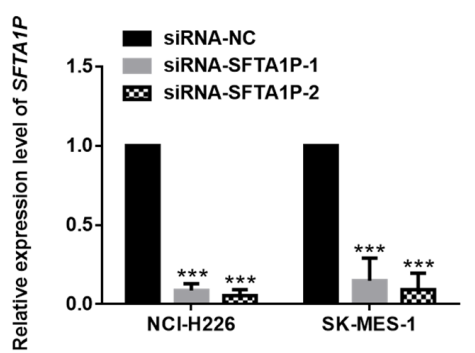

(E)

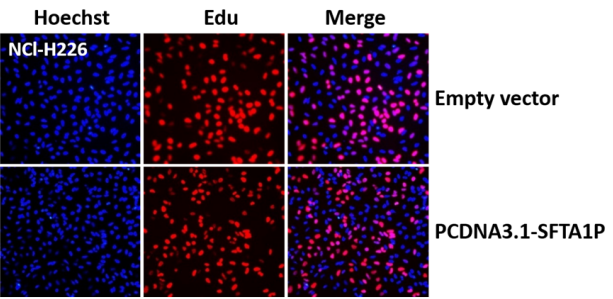

(G)

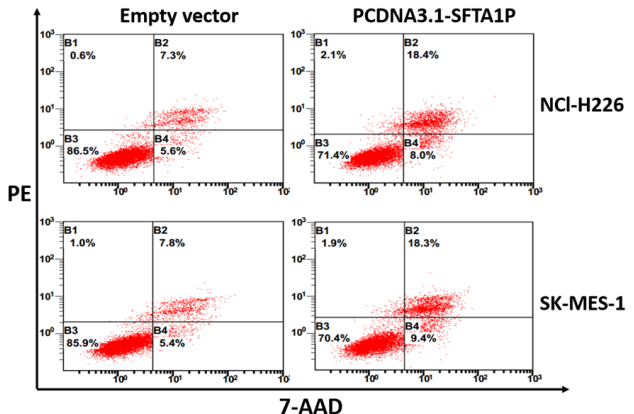

(B)

(D)
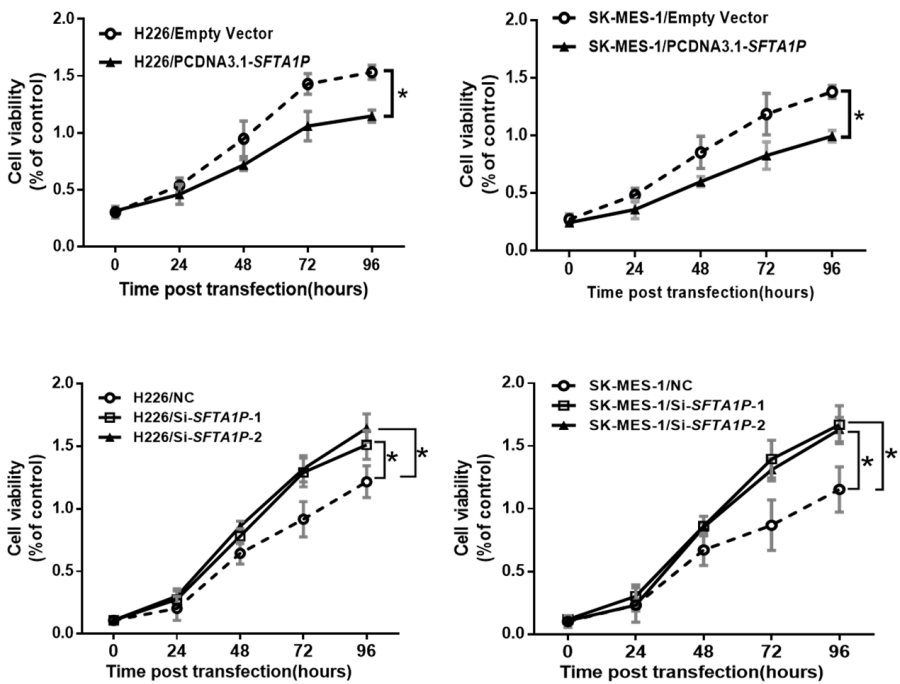

$(\mathrm{F})$
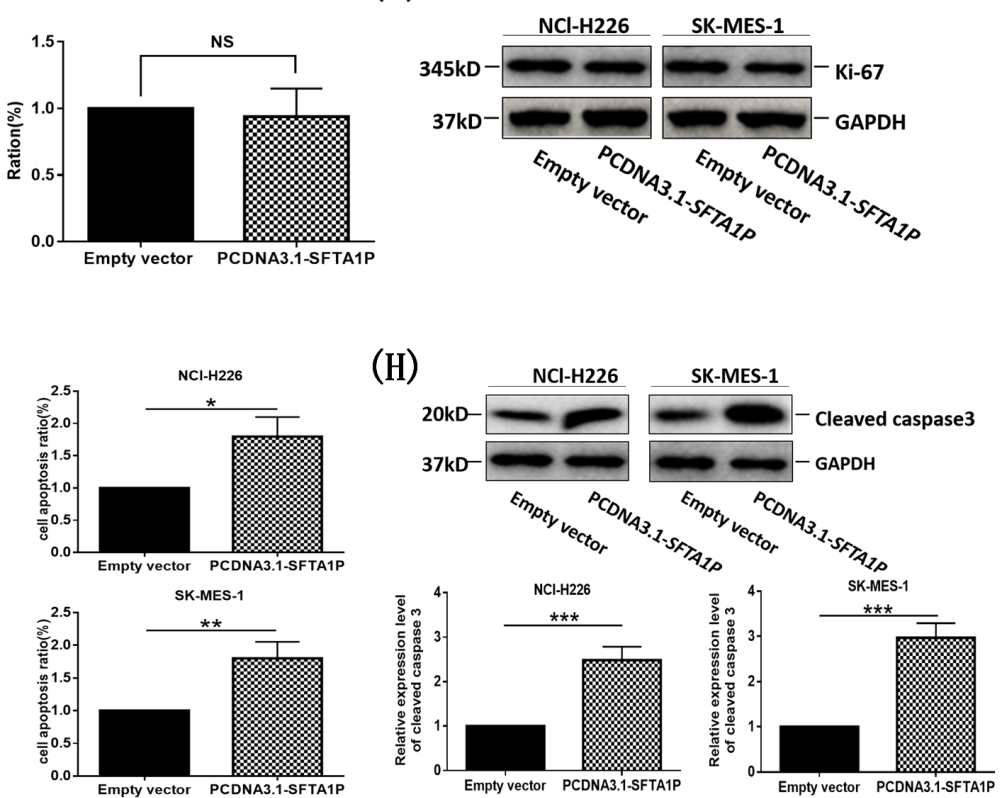

Figure 3: The cell viability and apoptosis induction in NCl-H226 and SK-MES-1 cells with elevated expression. The SFTA1P expression level was detected by qPCR after overexpression (Panel A) or knockdown (Panel C) SFTAlP 24h in NCl-H226 and SK-MES-1 cells. Panel B and Panel D. NCl-H226 and SK-MES-1 cells growth were measured by MTS assay. Panel E. The effect of SFTA1P on cell proliferation was measured by Edu staining assay in NCl-H226 cells. NCl-H226 cells were cultured in 96-well plates and transfected with $1 \mu \mathrm{g}$ PCDNA3.1-SFTA1P vector or empty vector for 24h, and then exposed to EdU (10uM) for 12h. Proliferating of $\mathrm{NCl}-\mathrm{H} 226$ cells were labeled with Edu (red) and DAPI (blue) to visualize the nuclei. The EdU-labeled replicating cells were examined under a fluorescent microscope. Data shown are representative images of individual groups ( $\mathrm{n}=6$ per group) from three independent experiments. Note that the number of NCl-H226 cells that incorporated EdU during transfected with PCDNA3.1(+)-SFTA1P vector did not significantly increase. Panel F. Western blot analysis of cell proliferation marker protein Ki-67 in SFTA1P-overexpression and control NCl-H226 and SK-MES-1 cells post transfection 48h. Panel G. 7-ADD/PE staining and flow cytometry analysis showed that SFTA1P overexpression in NCl-H226 and SK-MES-1 cells dramatically enhanced the cell apoptosis rate compared with control groups. Panel H. Western blot analysis of cell apoptosis marker protein cleaved caspase3 in SFTA1P-overexpression and control NCl-H226 and SK-MES-1 cells post transfection $48 \mathrm{~h}$. All experiments were performed in three biological replicates $(\mathrm{n}=3)$. And all data are shown as mean $\pm \mathrm{SD}$, ${ }^{*} \mathrm{p}<0.05,{ }^{* *} \mathrm{p}<0.01,{ }^{* * *} \mathrm{p}<0.001$. . 
(A)

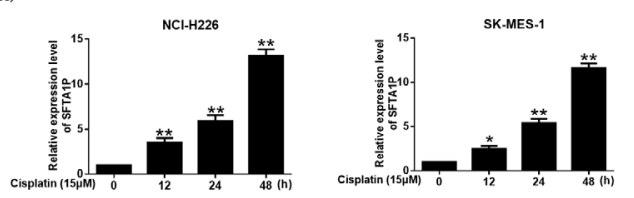

(B)

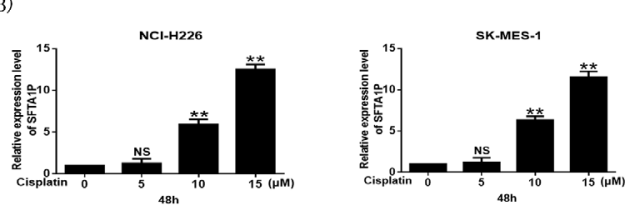

(C)

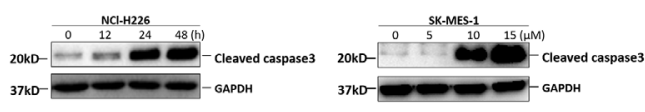

(E)
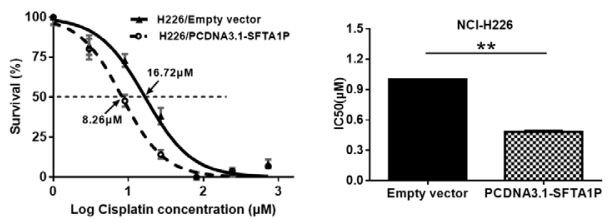

(G)

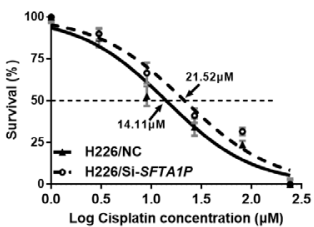

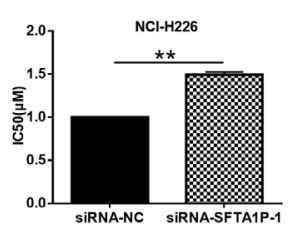

(D)

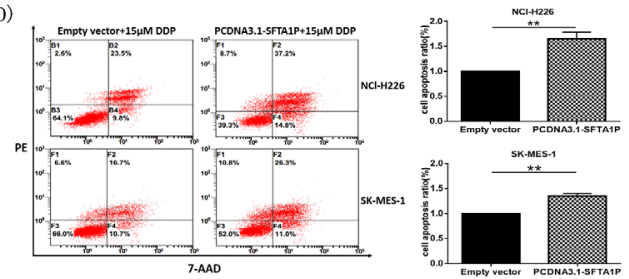

(F)
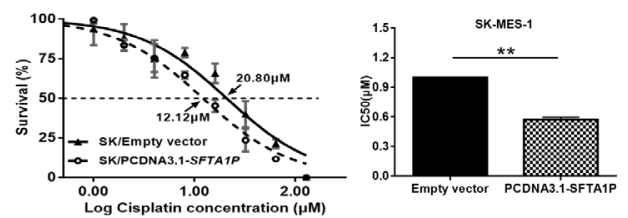

(H)
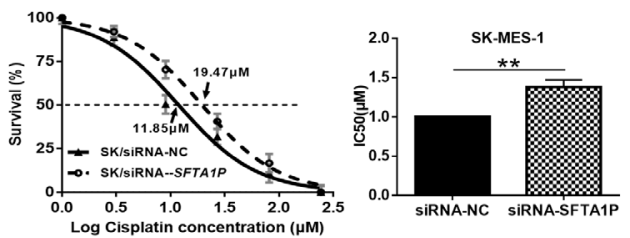
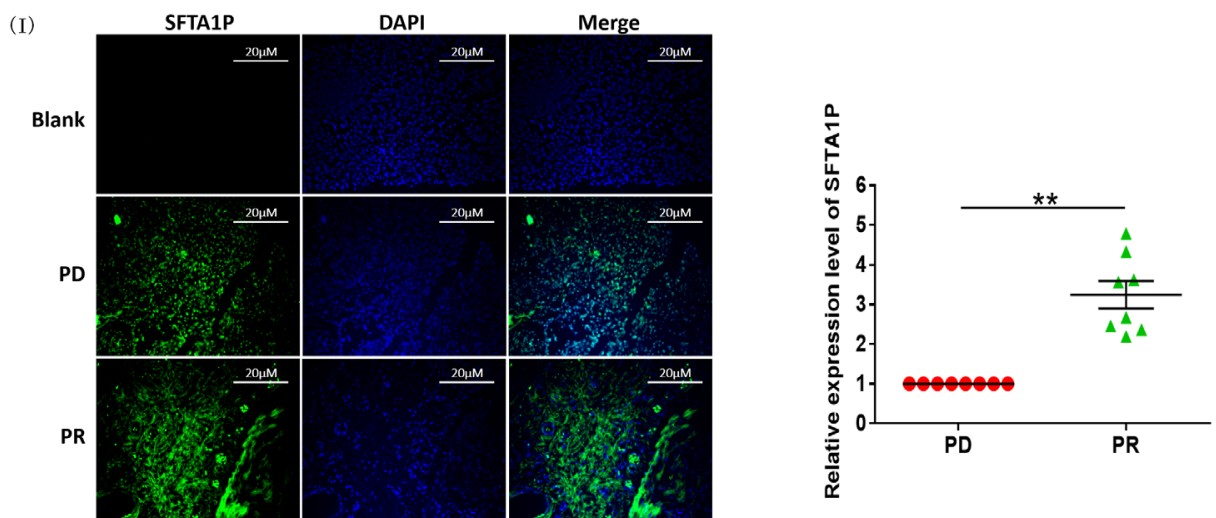

Figure 4: The cisplatin sensitivity was enhanced in LSCC cell lines and tissues while FTA1P was induced by cisplatin. Panel A. NCl-H226 and SK-MES-1 cells were treated with $15 \mu \mathrm{M}$ cisplatin for the indicated times. The mRNA level of SFTA1P was detected by q-RT-PCR. Panel B. NCl-H226 and SK-MES-1 cells were treated with cisplatin at the indicated doses for 48h, and then, the alteration of SFTA1P levels was analyzed by q-RT-PCR. Panel C. Apoptosis was detected by western blot analysis. After treated with cisplatin at the indicated doses and for the indicated times, $\mathrm{NCl}-\mathrm{H} 226$ cell lysates were analyzed by western blot analysis using the indicated antibodies. Panel D. Cell apoptosis was also evaluated using $7-\mathrm{ADD} / \mathrm{PE}$ staining with flow cytometry after the treatment with $15 \mu \mathrm{M}$ cisplatin for $24 \mathrm{~h}$ in NCl-H226 and SK-MES-1 cell lines. Representative flow cytometry results showing SFTA1P effects on cisplatin induced cell apoptosis in NCl-H226 and SK-MES-1 cells. Panel E-H. NCl-H226 and SK-MES-1 cells overexpressed or knockdown SFTA1P were treated with different concentrations of cisplatin $(1 \mu \mathrm{M}$ to $256 \mu \mathrm{M})$. $48 \mathrm{~h}$ later, cell viability was tested with an MTS assay. The half maximal inhibitory concentration $\left(\mathrm{IC}_{50}\right)$ was calculated from 3 independent experiments using GraphPad 5.0 software. The overexpression SFTA1P group exhibited a lower $\mathrm{IC}_{50}$ of cisplatin than the empty vector group. The data represent mean $\pm \mathrm{SD}$ of three independent experiments. " $\mathrm{p}<0.05$, ${ }^{* *} \mathrm{p}<0.01$. Panel I. RNA fluorescent in situ hybridization (FISH) analysis of SFTA1P expression in LSCC biopsy formalin-fixed paraffinembedded (FFPE) tissues with cisplatin chemotherapy curative effect evaluation $(\mathrm{n}=16)$. Cell nuclei are dyed DAPI shown in blue and SFTAIP-specific FISH shown in green. The first row represents the blank control, the second row represents the patients were resistance to cisplatin (PD), and the third row represents the patients were sensitive to cisplatin (PR). 
(A)

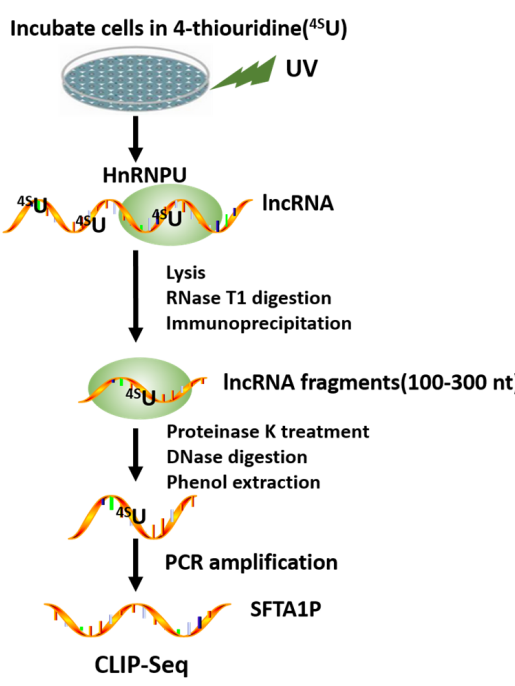

(E)

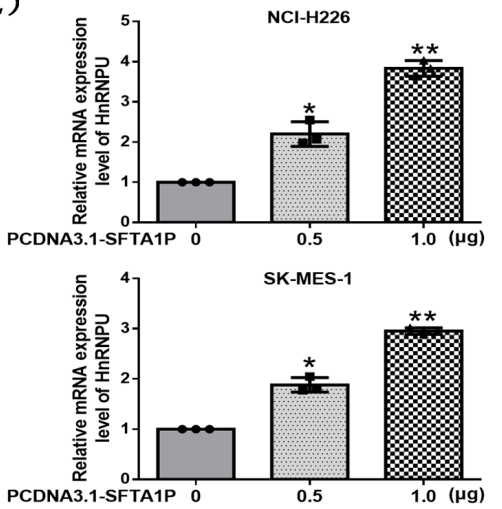

(B)

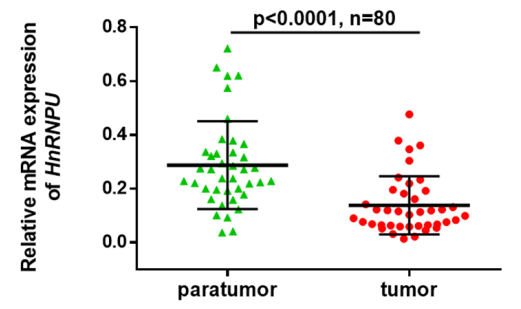

(D)

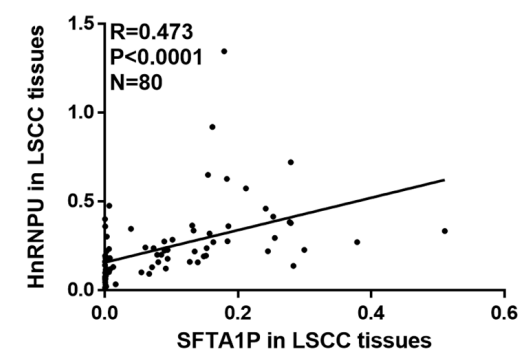

(F)
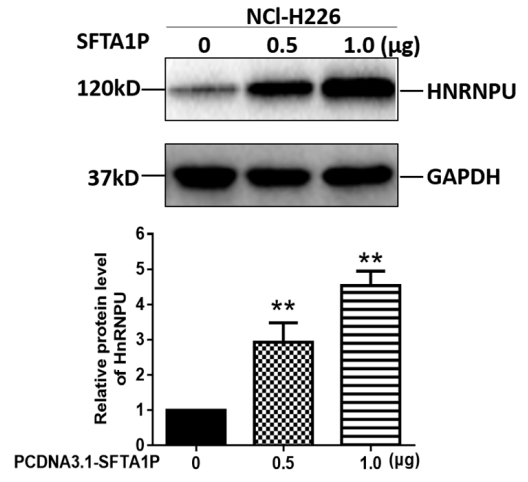

(C)
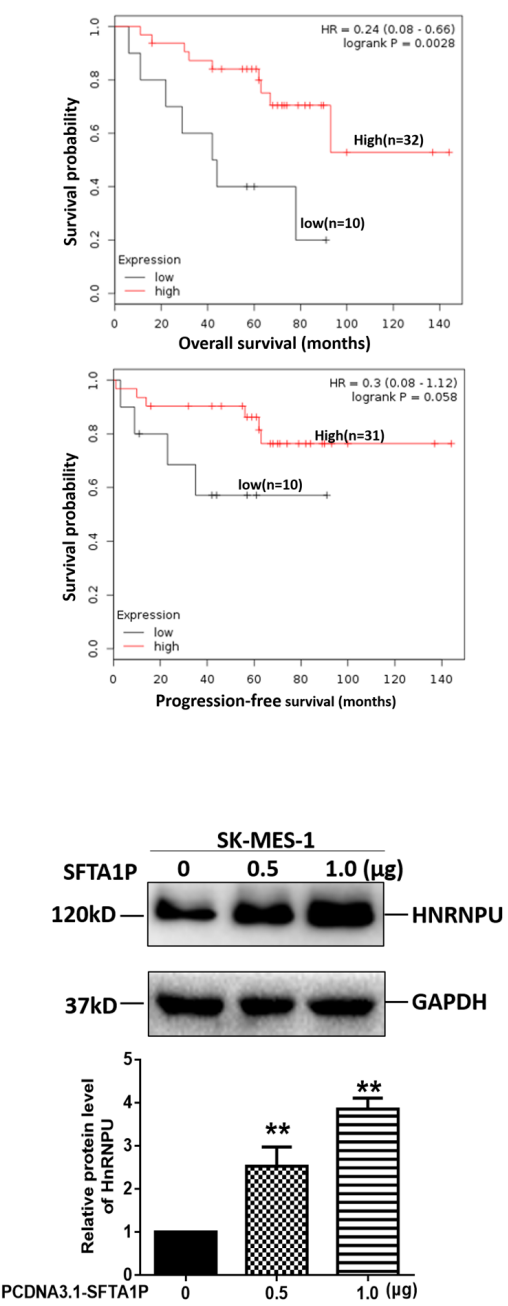

(G)

DAPI

HnRNPU

Merge
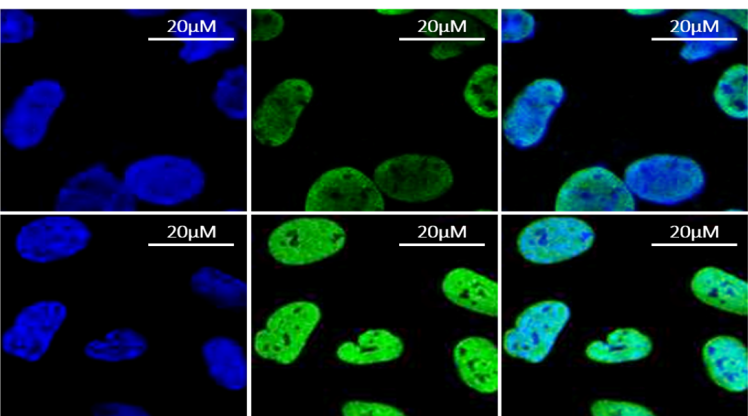

Empty vector

$\mathrm{NCl}-\mathrm{H} 226$

Figure 5: LncRNA SFTA1P upregulated the expression of hnRNP-U. Panel A. Schematic representation of the multiple steps of CLIP-seq. CLIP-seq analysis identified SFTAIP targets bound by hnRNPU (data from GSE34993). Panel B. Relative mRNA expression of hnRNPU in LSCC tissues detected by qRT-PCR. Panel C. Kaplan-Meier analysis of overall survival (OS) and progressionfree survival (PFS) in LSCC patients (data from GSE8894), and the median hnRNPU expression was used as a cutoff. It shows that high level of hnRNPU expression were correlated with a good OS and PFS. Panel D. The correlation between hnRNPU mRNA and SFTAIP expression in LSCC tumor tissues and para-tumor tissues $(\mathrm{n}=80)$. Panel $\mathbf{E}$ and Panel F. After respectively transfected $0.5 \mu \mathrm{g}$ and1.0 $\mathrm{\mu g}$ PCDNA3.1-SFTAlP vector for 24h or 48h, qRT-PCR and Western blot analysis were used to detecte the hnRNPU expression in NCl-H226 or SK-MES-1. Results are presented as mean $\pm \mathrm{SD},(\mathrm{n}=3) .{ }^{* *} \mathrm{p}<0.01$. Panel G. The immumofluorescence observation $(100 \times)$ of hnRNPU (green) in NCl-H226 cells after transfected with PCDNA3.1 (+)-SFTA1P plasmid or empty vector for 48h. It shows that hnRNPU located in the nucleus. And the overexpression SFTA1P group exhibited a higher expression of hnRNPU than the empty vector group. 
progression-free survival (PFS) than those with low hnRNP-U expression in GSE50081 dataset (Figure 5C). Moreover, we observed a significantly positive correlation between the mRNA level of hnRNP-U and SFTA1P transcriptional level in LSCC tissues (Figure 5D), which further suggests that upregulation of SFTA1P may lead to elevated expression of hnRNP-U in LSCC.

To determine the effect of SFTA1P on hnRNP-U expression, we detected hnRNP-U mRNA and protein alteration in NCl-H226 and SK-MES-1 cell lines transfected with SFTA1P overexpressing vector. The results showed that SFTA1P expression significantly increased hnRNP-U mRNA and protein levels in a dosedependent manner (Figure 5E and 5F). This phenomenon was also observed by immune-fluorescence assay (Figure $5 \mathrm{G})$.

\section{LncRNA SFTA1P and hnRNP-U expression were correlated with GADD45A expression in LSCC cells and tissues}

HnRNP-U is an abundant nuclear protein. It belongs to the subfamily of ubiquitously expressed heterogeneous nuclear ribonucleoproteins (hnRNPs). It was essential for cell viability [27], RNA stability control [20], and DNA damage response $[23,28]$.

To investigate the biological functions of hnRNP-U in the cisplatin sensitivity of LSCC, we performed MTS assay to examine the effect of hnRNP-U on cell sensitivity to cisplatin in NCl-H226 and SK-MES-1 cells. Cells were transfected with hnRNP-U-specific siRNA and the protein expression of hnRNP-U was detected by western blot (Figure 6A). $\mathrm{IC}_{50}$ value for cisplatin after hnRNP-U down-regulation was measured. Compared with NCl-H226 (or SK-MES-1) cells transfected with siRNA$\mathrm{NC}$, the $\mathrm{IC}_{50}$ value for cisplatin in NCl-H226 (or SKMES-1) transfected with siRNA-hnRNP-U-1 and siRNAhnRNP-U-2 was significantly increased (Figure 6B). While co-transfected PCDNA3.1-SFTA1P and siRNAhnRNP-U-2 in LSCC cell lines, the effect of SFTA1P induced sensitivity to cisplatin was abolished (Figure 6B). Together, these data suggest that SFTA1P induces apoptosis and increases LSCC cells sensitivity to cisplatin through upregulating hnRNP-U expression.

A previous study has shown that hnRNP-U can enhance the expression of growth arrest and DNA damage-45 alpha (GADD45A) by stabilizing its mRNA [20]. GADD45A is often up-regulated in response to various environmental stresses and drug therapies [29, 30]. It has been shown that GADD45A plays a role in inhibiting cell growth [31], promoting apoptosis [32], participating in DNA repair, and cancer cell survival.

We further identified the underlying mechanism by which hnRNP-U regulates GADD45A expression using qPCR and WB. As shown in Figure 6C, after hnRNP-U knockdown, GADD45A mRNA and protein expression was significantly down-regulated. Meanwhile, our correlation analysis found that SFTA1P and hnRNP-U expression was positively correlated with GADD45A level in 80 LSCC tissues (Figure 6D). Further KaplanMeier survival analysis illustrated that patients with low GADD45A expression had notably shorter overall survival (OS) (Figure 6E). These results suggest that SFTA1P and hnRNP-U contributed to cisplatin sensitivity in LSCC via induction of GADD45A expression.

\section{DISCUSSION}

Cisplatin is an important drug used in LSCC chemotherapy [3, 4]. However, the chemoresistance impedes its clinical usage [33, 34]. Chemoresistance remains a major obstacle in LSCC chemotherapy. Therefore, a better understanding of the underlying molecular mechanisms of tumourigenesis and chemoresistance is urgently required for improving LSCC treatment.

LncRNAs are a class of ncRNAs with transcript size $>200$ nucleotides [35]. They resemble mRNAs structurally but display a highly tissue-specifc expression pattern [36]. So, IncRNAs provide an opportunity to identify both cancer-specific biomarkers and functional drivers for lung cancer. Accumulating studies have demonstrated that lncRNAs play an important role in tumourigenesis and chemoresistance. For example, long noncoding RNA CCAT1 was reported to act as an oncogene and promote chemoresistance in docetaxel-resistant lung adenocarcinoma cells [37]; IncRNA FENDRR may act as an inhibitory molecule of doxorubicin-resistance through down-regulating the expression of $\mathrm{ABCB} 1$ and $\mathrm{ABCC} 1$ genes in osteosarcoma cells [38]; long non-coding RNA LINC00161 has been showed to sensitize osteosarcoma cells to cisplatin-induced apoptosis by regulating the miR-645-IFIT2 axis [39]. However, our knowledge of tumourigenesis and cisplatin chemosensitivity related lncRNAs in LSCC is still limited.

In our previous study, we investigated the genomewide expression profile of lncRNAs in 16 LSCC tumors and matched adjacent normal lung tissues by microarray. A total of 852 down- and 2,748 up-regulated probes were identified to be significantly differentially expressed in tumor tissues [18]. Among these, we identified that SFTA1P expression was significantly decreased in LSCC tumor tissues compared with matched adjacent normal lung tissues (Figure 2A) and was correlated with poor prognosis (Figure $2 \mathrm{D}$ and $2 \mathrm{E}$ ). Furthermore, we found that lncRNA SFTA1P expression was highly tissue specific in lung (Figure $1 \mathrm{~F})$, and it was downregulated in cisplatin-resistant cells (A549-DDP) (Figure 2C). Similar results were obtained in cisplatin-resistant LSCC patient's tumor tissues (Figure 4I). In addition, we found that SFTA1P could be induced by cisplatin (Figure 4A and 4B). We further used gene intervention technology to overexpress SFTA1P and found that cell apoptosis and sensitivity to cisplatin was markedly 
increased, and vise verse. Overexpression of SFTA1P can improve the sensitivity of LSCC cells to cisplatin, which demonstrated that SFTA1P was possibly involved in chemoresistance and may serve as a biomarker to predict the chemo-response and prognosis of LSCC patients (Figures $3 \mathrm{G}$ and $4 \mathrm{D}-4 \mathrm{H})$. The molecular mechanism that leads to the altered levels of SFTA1P during cisplatin treatment will be investigated in future.

Subsequently, we explored the potential mechanism of SFTA1P in response to cisplatin treatment in LSCC. By analyzing the crosslinking and immunoprecipitation combined with high-throughput
(A)

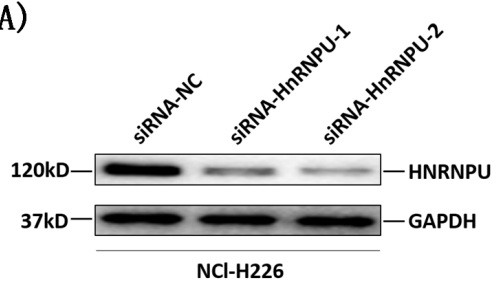

(B)
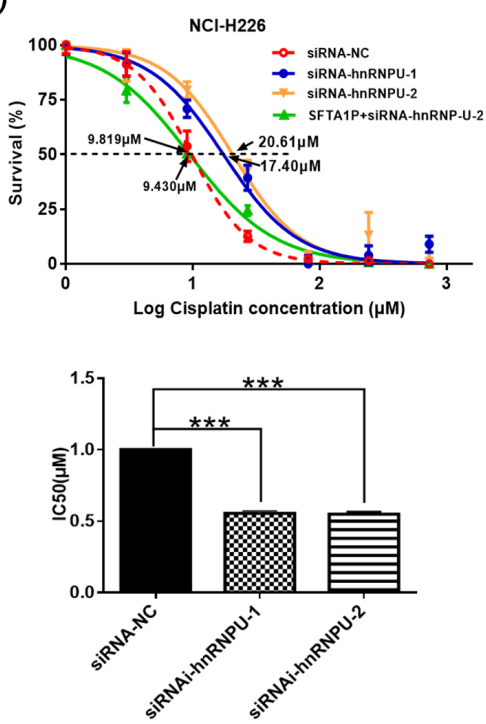

(C)
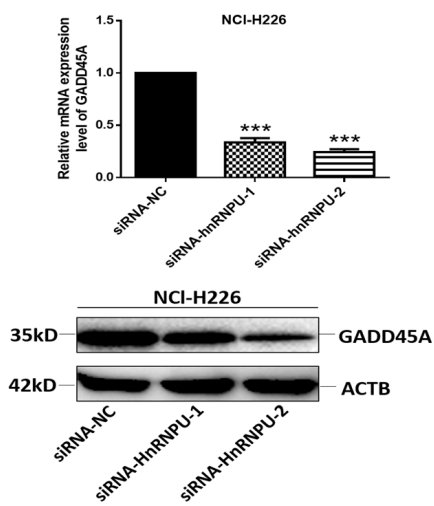
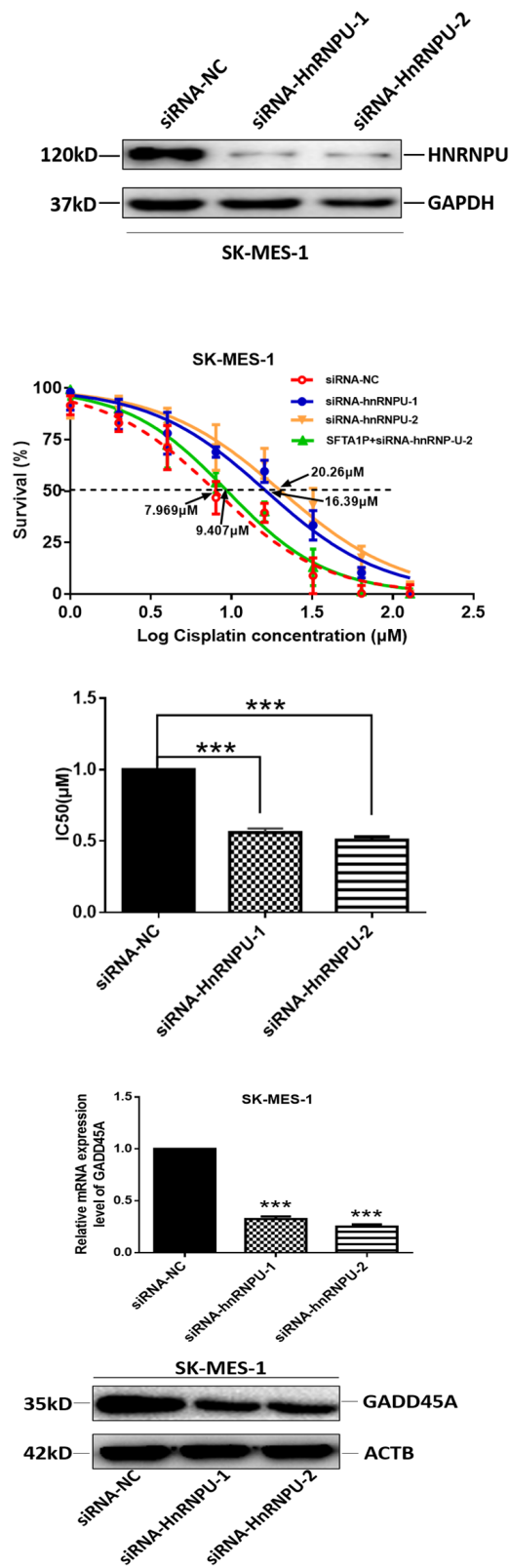

(D)
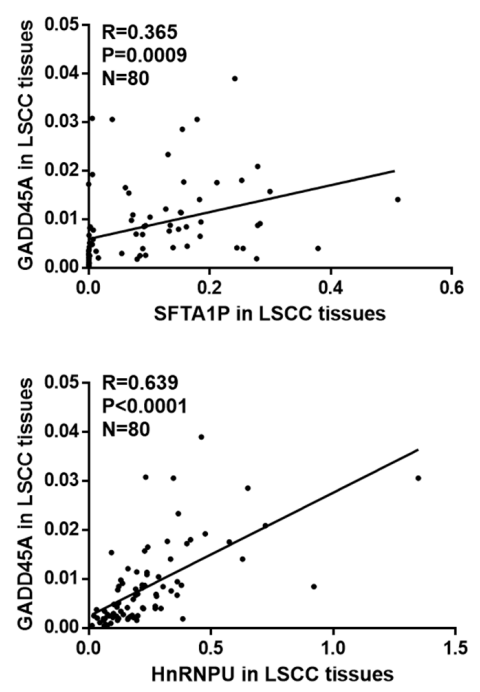

(E)

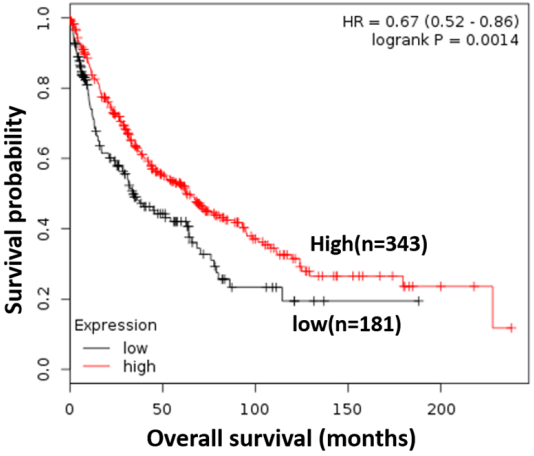

Figure 6: LncRNA SFTA1P and hnRNP-U expression were correlated with GADD45A expression in LSCC cells and tissues. Panel A. Knockdown of hnRNPU was checked by Western blot in NCl-H226 andSK-MES-1 cells after transfection 24h. Panel B. After transfected 24h, NCl-H226 and SK-MES-1 cells were treated with different concentrations of cisplatin, and then cell viability was tested with an MTS assay. 48h later, the half maximal inhibitory concentration $\left(\mathrm{IC}_{50}\right)$ was calculated from 3 independent experiments using GraphPad 5.0 software. The data represent mean \pm SD of three independent experiments. ${ }^{* *} p<0.001$. Panel C. After knockdown hnRNPU $24 \mathrm{~h}$ or $48 \mathrm{~h}$, the mRNA and protein of GADD45A were detected by qRT-PCR and Western blot analysis. Panel D. The correlation between hnRNPU, GADD45A mRNA and SFTA1P expression in LSCC tumor tissues and para-tumor tissues (n=80). Panel E. Kaplan-Meier analysis of overall survival (OS) in LSCC patients (http://kmplot.com/analysis/index.php? $\mathrm{p}=$ service\&start=1), and the median GADD45A expression was used as a cutoff. It shows that high level of GADD45A expression was correlated with a good OS. 
sequencing (CLIP-seq) data (from GSE34993), we found that SFTA1P could bind with hnRNP-U. HnRNP-U has been reported to play a crucial role in various pathological and physiological processes such as cell viability [27], RNA stability control [20], and DNA damage response $[23,28]$. In the current study, we found that SFTA1P increased cisplatin sensitivity through upregulating the expression of hnRNP-U in LSCC (Figures $5 \mathrm{E}-5 \mathrm{G}$ and $6 \mathrm{~B}$ ). However, the underlying regulatory mechanism remains unclear.

It is well known that lncRNAs molecular function depends on their subcellular localization. In this study, we detected the subcellular localization of SFTA1P by FISH assay and cytoplasmic and nuclear RNA fractionation analysis, we found that SFTA1P was mainly located in the nucleus (Figure 1D and 1E). It is widely accepted that lncRNAs locating in nucleus have regulatory roles in gene expression at the transcriptional levels. According to the experiment results, we suspect that SFTAIP regulated the hnRNP-U expression transcriptionally, but the specific regulation mechanisms still need to be further validated.

It has shown that hnRNP-U can enhance the expression of GADD45A by stabilizing mRNA [20]. GADD45 is involved in the DNA damage repair [31], and promoting apoptosis [32, 40], it is also essential for cancer cell survival [32]. Consistently, we further found that the expression of SFTA1P was significantly positively correlated with hnRNP-U and GADD45A, and high expression of hnRNP-U, GADD45A contributed to longer OS in LSCC patients. Further PCR and WB assays showed that SFTA1P enhanced hnRNP-U expression and promoted apoptosis. On the basis of the previously reported mechanism by which hnRNP-U regulates gene expression, we hypothesized that it promoted the expression of GADD45A. And it was validated by down-regulation of hnRNP-U (Figure 6). However, the specific regulatory mechanism between lncRNA SFTA1P and these two drug resistance related genes needs to be further studied by RNA pull down and chromatin immunoprecipitation.

In summary, we have shown that lncRNA SFTA1P and hnRNP-U were down-regulated in LSCC tissues and correlated with poor prognosis in LSCC patients. Both of them are involved in the progression of cisplatin sensitivity in LSCC. SFTA1P expression could be induceed by cisplatin and it promoted apoptosis and enhanced cisplatin sensitivity in LSCC by upregulating the expression of hnRNP-U at both the mRNA and protein levels. Moreover, SFTAIP facilitated the DNA damage repair and apoptosis related gene GADD45A expression. Our results indicate that IncRNA SFTA1P sensitizes LSCC cells to cisplatin-induced apoptosis by regulating the hnRNP-U-GADD45A axis, and it might be a candidate prognostic biomarker and a target for reversing cisplatin resistance in LSCC.

\section{MATERIALS AND METHODS}

\section{Tissue specimens' collection}

We obtained 80 LSCC tissues and paired adjacent non-tumor lung tissues (NTL) from patients who underwent surgery and 16 formalin-fixed paraffinembedded (FFPE) tissues from patients that were diagnosed with LSCC based on histopathological evaluation at Xiangya Hospital of Central South University (Changsha, Hunan, China). All of them provided written informed consents, in compliance with the code of ethics of the World Medical Association (Declaration of Helsinki) at the time of surgery for the donation of their tissues for this research. This project's protocol was approved by the Ethics Committee of Xiangya School of Medicine, Central South University (registration number of CTXY-110008-3). All fresh tissues were instantly frozen in liquid nitrogen after resection and stored at $-80^{\circ} \mathrm{C}$ until use, and the FFPE tissues were stored in a cool, dry place at room temperature.

\section{Cell culture and reagents}

Five non-small cell lung cancer cell lines (NCl-H226, SK-MES-1, NCl-H1299, A549, A549-DDP (cisplatin resistance cell line)) and a normal bronchial epithelial cell line HBE were obtained from the Institute of Biochemistry and Cell Biology of the Chinese Academy of Sciences (Shanghai, China). NCl-H226, NCl-H1299, A549 and A549-DDP cells were cultured in RPMI-1640 containing $10 \%$ fetal bovine serum (FBS, Gibico-BRL), and HBE, SK-MES- 1 cells were cultured with DMEM and MEM, respectively. Medium was renewed every day and cells were passaged before reaching confluence.

\section{RNA extraction and real-time RT-PCR}

To isolate cytoplasmic and nuclear RNAs from cells, we used a PARIS kit (Ambion) and followed the manufacturer's instruction. Total RNA was isolated using Trizol (Invitrogen) from tissue samples or cells according to the manufacturer's instructions. One microgram of total RNA was reverse transcribed in a final volume of $20 \mu \mathrm{l}$ using a PrimeScriptTM RT reagent kit (Takara, RR091A) according to the manufacturer's instructions. Quantitative reverse transcriptase polymerase chain reaction (qRTPCR) was performed in triplicate on the LC480, and data were calculated using the $2^{-\Delta \Delta C T}$ method. All primer sequences were listed in Supplementary Table 1.

\section{Cell transfection}

Overexpression: The human SFTAlP complementary DNA (cDNA) sequence was synthesized and then subcloned into the pCDNA3.1 $(+)$ vector (GeneChem, Shanghai, China) to generate the pCDNA3.1 
(+)-SFTA1P vector. Empty vector was used as a control. Knockdown: Silencing of SFTAIP was achieved by specific siRNAs. Three SFTA1P-specific siRNAs were synthesized by Ribobio (Guangzhou, China), the siRNA sequences were listed in Supplementary Table 1. A scrambled siRNA was used as negative control (NC). NCl-H226, SK-MES-1 cells were transfected with pCDNA3.1 (+)-SFTA1P vector, empty vector and siRNAs using Lipofectamine 2000 (Invitrogen, Carlsbad, CA, USA) according to the manufacturer's directions, and the cells were incubated for $24 \mathrm{~h}$ before experimental use.

\section{Drug sensitivity assay}

Cells were seeded in 96-well plates at a proper density of 4000 cells per well and were incubated overnight at $37^{\circ} \mathrm{C}$. The cells were incubated with different concentrations of cisplatin for $48 \mathrm{~h}$ at $37^{\circ} \mathrm{C}$. After addition of $20 \mu \mathrm{l}$ of Cell Titer $96^{\circledR}$ AQueous One Solution Cell Proliferation Assay (MTS) (Promega) to each well, plates were incubated for $1 \mathrm{~h}$ at $37^{\circ} \mathrm{C}$. Absorbance of each well at 490nm (A490) was detected using a spectrophotometer. The concentration of each drug that gives rise to $50 \%$ inhibition of growth $\left(\mathrm{IC}_{50}\right)$ was estimated from relative survival curves. Three independent experiments were performed in six duplicate wells. Cisplatin was obtained from Sigma-Aldrich (St. Louis, MO, USA, P4394).

\section{EdU incorporation assay}

The impact of overexpressing SFTA1P on NCl-H226 and SK-MES-1 cells proliferation was measured by 5-ethynyl-20-deoxyuridine (EdU) incorporation assay using an EdU assay kit (Cat.No:C10310, RiboBio, China). 24h after transfection, cells were incubated with culture medium supplemented with $10 \mu \mathrm{M}$ EdU for $12 \mathrm{~h}$. And then, cells were treated following the manufacturer's instruction.

\section{Apoptosis detection by flow cytometry assay}

NCl-H226 and SK-MES-1 cells were seeded in sixwell plates. After growing for $12 \mathrm{~h}$, cells were transiently transfected with the PCDNA3.1-SFTA1P or empty vector and harvested by trypsinization $48 \mathrm{~h}$ later, or treated with $15 \mu \mathrm{M}$ cisplatin for $24 \mathrm{~h}$ followed by resuspension in complete medium. Next, cells were washed twice with cold PBS and resuspended in 1X Binding Buffer at a concentration of $1 \times 10^{6}$ cells $/ \mathrm{ml}$. Then, cells were treated following the manufacturer's instruction of PE Annexin V Apoptosis Detection Kit I (BD, Cat. NO: 559763). The procedures were described as follows: Transfer $100 \mu$ of the solution $\left(1 \times 10^{5}\right.$ cells $)$ to a $5 \mathrm{ml}$ culture tube, then add $5 \mu \mathrm{l}$ of $\mathrm{PE}$ Annexin $\mathrm{V}$ and $5 \mu \mathrm{l} 7 \mathrm{AAD}$, gently vortex the cells and incubate for $15 \mathrm{~min}$ at $\mathrm{RT}\left(25^{\circ} \mathrm{C}\right)$ in the dark, finally add $400 \mu$ of $1 \mathrm{X}$ Binding Buffer to each tube. Analyze by flow cytometry within $1 \mathrm{~h}$.

\section{RNA fluorescence in situ hybridization (FISH)}

The SFTA1P cDNA probes were designed and synthesized by BersinBio (Guangzhou, China). These probes (probe A: 1-TTCCCAGGGCGCTCTCTGCCAA G-23; probe B: 390-TTTCCATCCTGAGGT GGTCTGCC-412; probe C: 849-AAG ATGAATGTAAGGTTTTATTG-871) were labeled by 5'-FAM. Then, the RNA FISH experiments were performed on NCl-H226, A549 cells and on paraffin embedded sections of 8 LSCC and their adjacent tissues. The RNA FISH was then carried out following the instruction of the FISH Detection Kit (BersinBio, Guangdong, China). Images were taken using a fluorescence microscope equipped with vision software. Detected signals were colored green (SFTA1P) and blue (DAPI). The fluorescence signals were quantified by image $\mathrm{J}$ software.

\section{Immunofluorescence detection}

HnRNPU protein expression alteration in NCl-H226 cells was observed by immunofluorescence $48 \mathrm{~h}$ after cells were transfected with SFTA1P overexpressing vector. After growing for $12 \mathrm{~h}$, cells transiently transfected with the PCDNA3.1-SFTA1P or empty vectors were inoculated in special petri dish. After transfected for $48 \mathrm{~h}$, the cells were fixed with $4 \%$ paraformaldehyde, then washed in PBS at room temperature 3 times. Then the cells were permeabilized with $0.1 \%$ Triton X-100 (Beyotime) in PBS for 30min at room temperature, then washed in PBS three times. After incubated for $30 \mathrm{~min}, 1 \% \mathrm{BSA}$ incubated in PBST was used to block the binding of non-specific sites. 60 min later, primary antibody of hnRNPU (1:100, ab10297) in $1 \%$ BSA was added and the cells were incubated for $60 \mathrm{~min}$ at room temperature. Then $20 \mu \mathrm{l}$ fluorescence labeling antibody (1:50, Beyotime) was added and incubated for $30 \mathrm{~min}$. Nuclei were stained with DAPI (Cat. No A1001, Applichem, Germany) at a concentration of $10 \mathrm{ng} / \mathrm{ml}$ in cold dark place for $15 \mathrm{~min}$. Finally, cells were observed by fluorescent microscope.

\section{Western blot analysis}

$48 \mathrm{~h}$ after transfection, cells were collected and lysed using a RIPA lysis buffer that contained the protease inhibitor cocktail (Roche, Basel, Switzerland) and phenyl methyl sulfonyl fluoride (PMSF) (Roche). Cell protein lysates that contained $50 \mu \mathrm{g}$ protein were electrophoresed on $10 \%$ or $12 \%$ sodium dodecyl sulfate-polyacrylamide gel, and then transferred onto $0.22 \mathrm{~mm}$ or $0.45 \mathrm{~mm}$ PVDF membrane (Millipore, Bedford, MA). Then, the membranes were incubated in blocking solution ( $5 \%$ non-fat milk) for 
1.5 hour at room temperature (RT) and incubated with specific primary antibodies at $4^{\circ} \mathrm{C}$ overnight. The following antibodies were used in this study: GAPDH (Sigma, G9545); ACTB (Beyotime, AA128); Cleaved Caspase-3 (Wanleibio, WL02348); Ki-67 (Wanleibio, WL01384a); GADD45A (Sigma, WH00016 47M1).

\section{Statistical analysis}

The statistical analyses were performed using SPSS 20.0 software (IBM, Inc., Chicago, IL, USA). The significance of differences between groups was estimated by the Student's t-test, or chi-square test. Pearson correlation analyses were conducted to investigate the correlation between SFTA1P and other gene expressions. Two-sided $p$ values were calculated, and a value of $\mathrm{P}<0.05$ was considered statistically significance. All values were presented as the mean $\pm \mathrm{SD}$.

\section{Author contributions}

L.L., J.Y.Y., and Z.Q.L. conceived and designed the experiments. L.L. and F.Z.H. performed the experiments and wrote the manuscript. L.L., M.S.H., Y.F.G., Y.X.C., D.B.Z., and W.Z. contributed clinical samples and data. X.C., L.Q.S., Z.Q.L. and H.H.Z. edited the manuscript.

\section{CONFLICTS OF INTEREST}

All authors declare that no conflicts of interest exist.

\section{FUNDING}

This work was supported by the National Key Research and Development Programs (2016YFC1306900, 2016YFC0905002), National Natural Science Foundation of China (81373490, 81573508, 81573463), The StrategyOriented Special Project of Central South University in China (ZLXD2017003), and the Natural Science Foundation of Hunan Provincial of China (2016JJ2154).

\section{REFERENCES}

1. Hammerman PS, Lawrence MS, Voet D, Jing R, Cibulskis K, Sivachenko A, Stojanov P, McKenna A, Lander ES, Gabriel S, Getz G, Sougnez C, Imielinski M, et al. Comprehensive genomic characterization of squamous cell lung cancers. Nature. 2012; 489:519-525.

2. Crino L, Weder W, van Meerbeeck J, Felip E. Early stage and locally advanced (non-metastatic) non-small-cell lung cancer: ESMO Clinical Practice Guidelines for diagnosis, treatment and follow-up. Ann Oncol. 2010; 21:v103-v115.

3. Scagliotti GV, Novello S, Rapetti S, Papotti M. Current state-of-the-art therapy for advanced squamous cell lung cancer. Am Soc Clin Oncol Educ Book. 2013:354-358.
4. Azzoli CG, Temin S, Aliff T, Baker SJ, Brahmer J, Johnson DH, Laskin JL, Masters G, Milton D, Nordquist L, Pao W, Pfister DG, Piantadosi S, et al. 2011 Focused update of 2009 American Society of Clinical Oncology Clinical Practice Guideline update on chemotherapy for stage IV non-smallcell lung cancer. J Clin Oncol. 2011; 29:3825-3831.

5. Schiller JH, Harrington D, Belani CP, Langer C, Sandler A, Krook J, Zhu J, Johnson DH. Comparison of four chemotherapy regimens for advanced non-small-cell lung cancer. N Engl J Med. 2002; 346:92-98.

6. Wang J, Zhang J, Zheng H, Li J, Liu D, Li H, Samudrala R, Yu J, Wong GK. Mouse transcriptome: neutral evolution of 'non-coding' complementary DNAs. Nature. 2004; 431:1757, discussion 757.

7. Struhl K. Transcriptional noise and the fidelity of initiation by RNA polymerase II. Nat Struct Mol Biol. 2007; 14:103-105.

8. Perkel JM. Visiting "noncodarnia". Biotechniques. 2013; 54:301, 303-304.

9. Matsumoto A, Pasut A, Matsumoto M, Yamashita R, Fung J, Monteleone E, Saghatelian A, Nakayama KI, Clohessy JG, Pandolfi PP. mTORC1 and muscle regeneration are regulated by the LINC00961-encoded SPAR polypeptide. Nature. 2017; 541:228-232.

10. Wang KC, Chang HY. Molecular mechanisms of long noncoding RNAs. Mol Cell. 2011; 43:904-914.

11. Mercer TR, Dinger ME, Mattick JS. Long non-coding RNAs: insights into functions. Nat Rev Genet. 2009; 10:155-159.

12. Bhartiya D, Kapoor S, Jalali S, Sati S, Kaushik K, Sachidanandan C, Sivasubbu S, Scaria V. Conceptual approaches for lncRNA drug discovery and future strategies. Expert Opin Drug Discov. 2012; 7:503-513.

13. Qu L, Ding J, Chen C, Wu ZJ, Liu B, Gao Y, Chen W, Liu F, Sun W, Li XF, Wang X, Wang Y, Xu ZY, et al. Exosometransmitted lncARSR promotes sunitinib resistance in renal cancer by acting as a competing endogenous RNA. Cancer Cell. 2016; 29:653-668.

14. Gong WJ, Yin JY, Li XP, Fang C, Xiao D, Zhang W, Zhou $\mathrm{HH}$, Li X, Liu ZQ. Association of well-characterized lung cancer lncRNA polymorphisms with lung cancer susceptibility and platinum-based chemotherapy response. Tumour Biol. 2016; 37:8349-8358.

15. Yan L, Yang M, Guo H, Yang L, Wu J, Li R, Liu P, Lian Y, Zheng X, Yan J, Huang J, Li M, Wu X, et al. Singlecell RNA-Seq profiling of human preimplantation embryos and embryonic stem cells. Nat Struct Mol Biol. 2013; 20:1131-1139.

16. Cabili MN, Trapnell C, Goff L, Koziol M, Tazon-Vega B, Regev A, Rinn JL. Integrative annotation of human large intergenic noncoding RNAs reveals global properties and specific subclasses. Genes Dev. 2011; 25:1915-1927.

17. Huarte $M$. The emerging role of lncRNAs in cancer. Nat Med. 2015; 21:1253-1261. 
18. Wang Y, Qian CY, Li XP, Zhang Y, He H, Wang J, Chen J, Cui JJ, Liu R, Zhou H, Xiao L, Xu XJ, Zheng Y, et al. Genome-scale long noncoding RNA expression pattern in squamous cell lung cancer. Sci Rep. 2015; 5:11671.

19. Fackelmayer FO, Richter A. hnRNP-U/SAF-A is encoded by two differentially polyadenylated mRNAs in human cells. Biochim Biophys Acta. 1994; 1217:232-234.

20. Yugami M, Kabe Y, Yamaguchi Y, Wada T, Handa H. hnRNP-U enhances the expression of specific genes by stabilizing mRNA. FEBS Lett. 2007; 581:1-7.

21. Xiao R, Tang P, Yang B, Huang J, Zhou Y, Shao C, Li H, Sun $\mathrm{H}$, Zhang Y, Fu XD. Nuclear matrix factor hnRNP U/SAF-A exerts a global control of alternative splicing by regulating U2 snRNP maturation. Mol Cell. 2012; 45:656-668.

22. Gohring F, Schwab BL, Nicotera P, Leist M, Fackelmayer FO. The novel SAR-binding domain of scaffold attachment factor A (SAF-A) is a target in apoptotic nuclear breakdown. EMBO J. 1997; 16:7361-7371.

23. Berglund FM, Clarke PR. hnRNP-U is a specific DNAdependent protein kinase substrate phosphorylated in response to DNA double-strand breaks. Biochem Biophys Res Commun. 2009; 381:59-64.

24. Lin MF, Jungreis I, Kellis M. PhyloCSF: a comparative genomics method to distinguish protein coding and noncoding regions. Bioinformatics. 2011; 27:i275-i282.

25. Krupp M, Marquardt JU, Sahin U, Galle PR, Castle J, Teufel A. RNA-Seq Atlas--a reference database for gene expression profiling in normal tissue by next-generation sequencing. Bioinformatics. 2012; 28:1184-1185.

26. Huelga SC, Vu AQ, Arnold JD, Liang TY, Liu PP, Yan BY, Donohue JP, Shiue L, Hoon S, Brenner S, Ares MJ, Yeo GW. Integrative genome-wide analysis reveals cooperative regulation of alternative splicing by hnRNP proteins. Cell Rep. 2012; 1:167-178.

27. Roshon MJ, Ruley HE. Hypomorphic mutation in hnRNP U results in post-implantation lethality. Transgenic Res. 2005; 14:179-192.

28. Britton S, Dernoncourt E, Delteil C, Froment C, Schiltz O, Salles B, Frit P, Calsou P. DNA damage triggers SAF-A and RNA biogenesis factors exclusion from chromatin coupled to R-loops removal. Nucleic Acids Res. 2014; 42:9047-9062.

29. Hirose T, Sowa Y, Takahashi S, Saito S, Yasuda C, Shindo N, Furuichi K, Sakai T. p53-independent induction of Gadd45 by histone deacetylase inhibitor: coordinate regulation by transcription factors Oct-1 and NF-Y. Oncogene. 2003; 22:7762-7773.

30. Yin F, Bruemmer D, Blaschke F, Hsueh WA, Law RE, Herle AJ. Signaling pathways involved in induction of
GADD45 gene expression and apoptosis by troglitazone in human MCF-7 breast carcinoma cells. Oncogene. 2004; 23:4614-4623.

31. Hollander MC, Fornace AJ. Genomic instability, centrosome amplification, cell cycle checkpoints and Gadd45a. Oncogene. 2002; 21:6228-6233.

32. Zerbini LF, Wang Y, Czibere A, Correa RG, Cho JY, Ijiri K, Wei W, Joseph M, Gu X, Grall F, Goldring MB, Zhou JR, Libermann TA. NF-kappa B-mediated repression of growth arrest- and DNA-damage-inducible proteins 45alpha and gamma is essential for cancer cell survival. Proc Natl Acad Sci U S A. 2004; 101:13618-13623.

33. Yang Y, Li H, Hou S, Hu B, Liu J, Wang J. The noncoding RNA expression profile and the effect of lncRNA AK126698 on cisplatin resistance in non-small-cell lung cancer cell. PLoS One. 2013; 8:e65309.

34. Liu MY, Li XQ, Gao TH, Cui Y, Ma N, Zhou Y, Zhang GJ. Elevated HOTAIR expression associated with cisplatin resistance in non-small cell lung cancer patients. J Thorac Dis. 2016; 8:3314-3322.

35. Ponting CP, Belgard TG. Transcribed dark matter: meaning or myth? Hum Mol Genet. 2010; 19:R162-R168.

36. Brunner AL, Beck AH, Edris B, Sweeney RT, Zhu SX, Li R, Montgomery K, Varma S, Gilks T, Guo X, Foley JW, Witten DM, Giacomini CP, et al. Transcriptional profiling of long non-coding RNAs and novel transcribed regions across a diverse panel of archived human cancers. Genome Biol. 2012; 13:R75.

37. Chen J, Zhang K, Song H, Wang R, Chu X, Chen L. Long noncoding RNA CCAT1 acts as an oncogene and promotes chemoresistance in docetaxel-resistant lung adenocarcinoma cells. Oncotarget. 2016; 7:62474-62489. https://doi.org/10.18632/oncotarget.11518.

38. Kun-Peng Z, Xiao-Long M, Chun-Lin Z. LncRNA FENDRR sensitizes doxorubicin-resistance of osteosarcoma cells through down-regulating $\mathrm{ABCB} 1$ and $\mathrm{ABCC} 1$. Oncotarget. 2017; 8:71881-71893. https://doi.org/10.18632/ oncotarget.17985.

39. Wang Y, Zhang L, Zheng X, Zhong W, Tian X, Yin B, Tian K, Zhang W. Long non-coding RNA LINC00161 sensitises osteosarcoma cells to cisplatin-induced apoptosis by regulating the miR-645-IFIT2 axis. Cancer Lett. 2016; 382:137-146.

40. Zerbini LF, Wang Y, Correa RG, Cho JY, Libermann TA. Blockage of NF-kappaB induces serine 15 phosphorylation of mutant $\mathrm{p} 53$ by JNK kinase in prostate cancer cells. Cell Cycle. 2005; 4:1247-1253. 